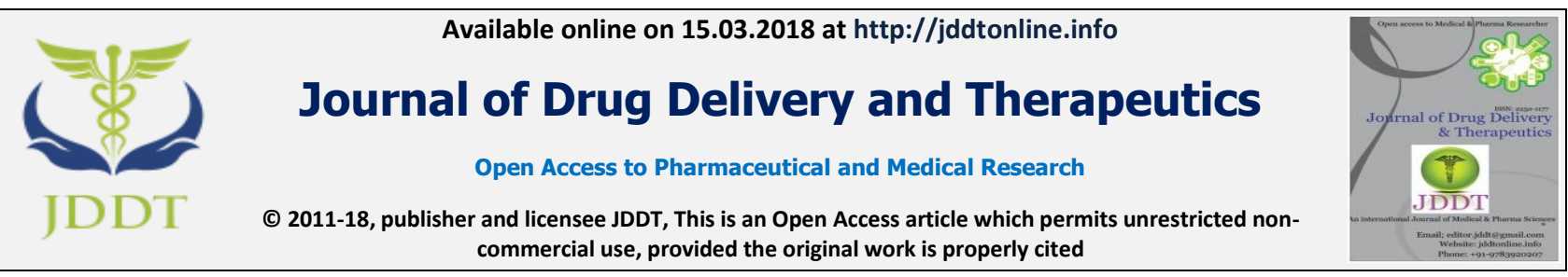

Open $\odot$ Access

Review Article

\title{
A REVIEW ON LEARNING AND MEMORY
}

\author{
Gupta Avneet*, Singh Manish Pal and Sisodia S. Siddhraj \\ Department of Pharmacology, Bhupal Nobles' College of Pharmacy, Bhupal Nobles' University, Udaipur, Rajasthan, India
}

\begin{abstract}
Learning is defined as the acquisition of information and skills and subsequent retention of the information is called memory. Dementia is one of the ages related mental problems and characteristic symptom of various neurodegenerative disorders including Alzheimer's disease which is age related. It is a progressive and neurodegenerative disorder. The analysis of the anatomical and physical bases of learning and memory is one of the great successes of modern neuroscience. The action of drugs on memory is more or less specific and serious depending on the memory system affected. So the present study is therefore focused on the various types of learning and memory.
\end{abstract}

Key Words: Learning, Memory, Dementia.

Article Info: Received 09 Jan, 2018; Review Completed 23 Feb, 2018; Accepted 25 Feb, 2018; Available online 15 March, 2018

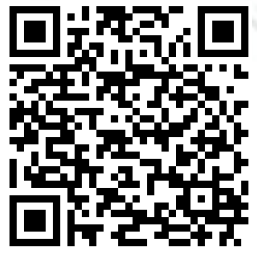

Cite this article as:

Gupta A, Singh MP, Sisodia SS, A review on learning and memory, Journal of Drug Delivery and Therapeutics. 2018; 8(2):153-157

DOI: http://dx.doi.org/10.22270/jddt.v8i2.1671

*Address for Correspondence

Avneet Gupta, Research Scholar, Department of Pharmacology, Bhupal Nobles' College of Pharmacy, Bhupal Nobles' University, Udaipur, Rajasthan, India.

\section{INTRODUCTION}

The analysis of the anatomical and physical bases of learning and memory is one of the great successes of modern neuroscience ${ }^{1}$. In this we will discuss different issues that are central to learning and memory. First what are the different types of learning and memory? Second, where in the brain is memory located and learning cycle. Third, different types of memory theories. Fourth, how memory work and disorders of memory. Learning is the process by which we acquire knowledge about the world. While this definition is erudite, it doesn't help us much in knowing what to study $^{2}$. Another definition Learning refers to a more or less permanent change in behavior which occurs as a result of practice, is a little better ${ }^{3}$. Learning and memory are vital attributes of human intelligence. These processes underlie the very nature of our selfawareness ${ }^{4}$. Learning is a change in behavior or in potential behavior that occurs as results of experience. The three major types of learning described by behavioral psychology are classical conditioning, operant conditioning and observational learning 5 . When people identify objects in the environment (e.g. knowing that a thing is a plant or animal) or when they give the answer to a factual question, they draw upon stores of general knowledge about the world accumulated over time. This type of memory is often referred as a sematic memory ${ }^{6}$. The action of drugs on memory is more or less specific and serious depending on the memory system affected. Thus, analysis of the type of memory alteration can be used to incriminate more a particular drug during polymedication. Dementia is one of the ages related mental problems and characteristic symptom of various neurodegenerative disorders including Alzheimer's disease which is age related. It is a progressive and neurodegenerative disorder which primarily affects the elderly population and it is considered to be responsible for $60 \%$ of all dementia in people who are aged 65 or more. It is debilitating in nature and due to which an enormous social and economic worry is placed on our society. Currently there is no proper cure for the disorder and much of the treatments available have been able to only delay the progression of the disease or provide 
symptomatic relief for a short time period. Therefore there is a need for a different approach to the treatment of these diseases ${ }^{7}$. The present study is therefore the focused on discussing the various types of learning and memory.

\section{TYPES OF LEARNING}

\section{Associative Learning}

In associative learning, we "learn" that two stimuli are associated with each other or that a response is associated with a given event or have a given consequence. Perhaps important in clinical considerations, a person can also learn that an outcome is not associated with a response. So a person may learn that what happens to him is not related to what he does $^{8}$. It is the process by which someone learns an association between two stimuli or behavior and a stimulus Associative learning occurs when you learn something based on a new stimulus ${ }^{9}$.

\section{Classical Conditioning}

Classical conditioning is well demonstrated by Pavlov's famous experiment in which he presented meat powder to a dog, causing it to salivate. He repeated the presentation, and each time the dog salivated. If he repeatedly rang a bell just before presenting the meat powder (they were paired), the animal came to associate the bell with the presentation of the meat powder, and it would begin to salivate when the bell was rung. In fact, for a while it would salivate if the bell was rung but no meat powder was presented (they were unpaired). After a while, the bell stopped predicting the presentation of meat powder for the dog and it ceased salivating when it was rung. This process is called extinction ${ }^{\mathbf{1 0}}$.

It should be noted that for classical conditioning to occur the ringing of the bell must precede the presentation of the meat powder often by a certain critical interval of time (of the order of $0.5 \mathrm{sec}$ ). One way to look at classical conditioning is to think of the bell as becoming a signal that the meat powder is about to be presented. In Pavlov's paradigm, the meat powder normally elicits salivation without experimenter intervention (it is innate or perhaps previously strongly learned) and it is called the unconditioned stimulus (US). The response is called the unconditioned response (UR). The bell comes to elicit salivation only after it is repeatedly paired with meat powder; so it's called the conditioned stimulus (CS). The response to it (again salivation) is called the conditioned response (CR). The UR and the $\mathrm{CR}$ are usually similar but often not identical in type or strength $^{11}$.

\section{Before Conditioning}

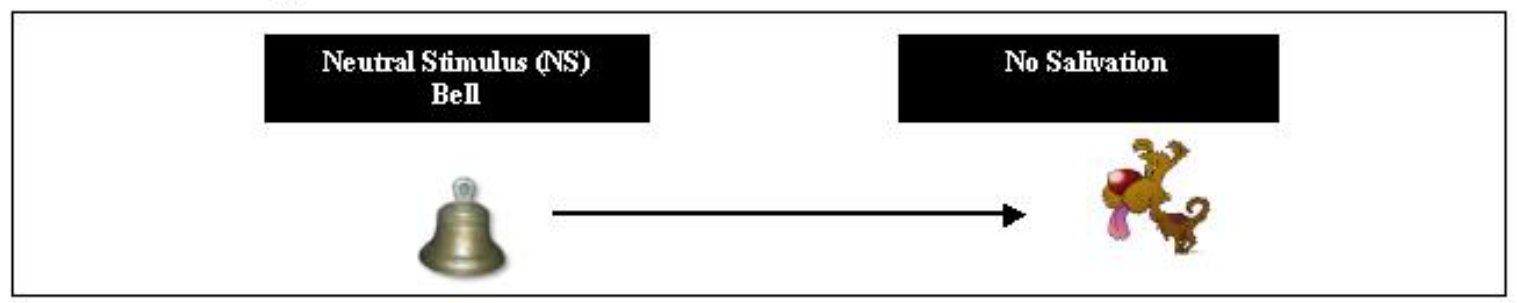

During Conditioning

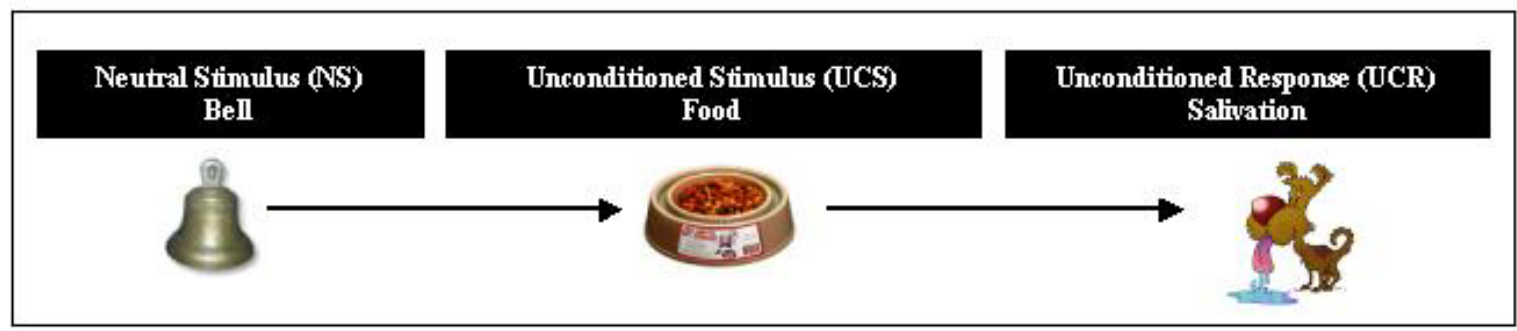

After Conditioning

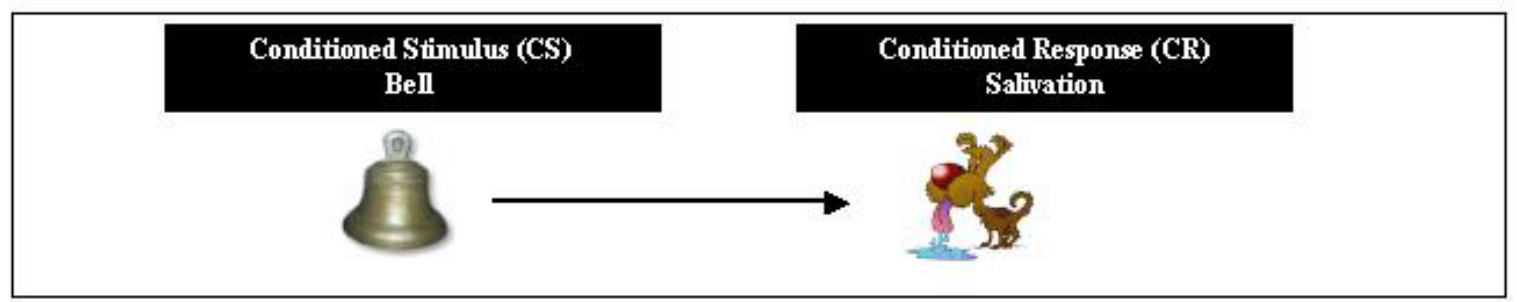

Figure 1: Classical Conditioning: Learning through Association.

\section{Operant Conditioning}

Operant conditioning from psychologist B.F. Skinner's experiments, involves the use of a schedule of reinforcements or rewards and punishments until the behavior is learned. For instance, if the dog were to hear the bell and step on a lever, it would receive the dog biscuit the reward. Alternatively, if the dog were to step on the lever when the bell doesn't ring and receive a 
shock - a positive punishment that would shape behavior in the opposite direction. A negative punishment, by contrast, would be to take away something, such as a biscuit, if the dog barks. If a child completes the home work she is praised (rewarded) by the parents and the child learns to perform the task. If the child breaks a plate, he/she is scolded (punished) and she will learn not to repeat the behavior. This is called Operant Conditioning or instrumental conditioning ${ }^{\mathbf{1 2}}$. In other words, we learn to perform behaviors that produce positive outcomes and avoid behaviors that yield negative outcomes. We may define operant conditioning as a process through which organisms learn to repeat behaviors that produce positive outcomes or avoid or escape from the negative outcomes. B.F. Skinner is considered as the most influential psychologist advocating the role of operant conditioning in learning. He developed an experimental chamber (called Skinner box) to study learning process in rats. The chamber included a lever attached to the front wall. Pressing the lever is the response to be learned. The hungry rat is placed in the chamber and it starts doing random activity in it. After some time, the rat accidentally presses the lever and a pellet of food drops automatically in the plate and the rat eats it. After eating the pallet the rat again starts activity in the chamber. After some activity it again presses the lever and gets pellet (a reward). Gradually the random activity changes to more specific activity around the lever. Finally, the rat learns that pressing the lever results in dropping of the food, a satisfying outcome. In other words the pressing of lever by the rat is instrumental in providing food (reinforcement). The response (pressing the lever) is reinforced and the behavior is acquired or learned. The pressing of lever by the rat is instrumental in getting the food, a satisfying consequence (positive reinforcement) and that is why this type of learning is also called instrumental learning. It is also called operant conditioning because the behavior of rat or any organism is a kind of operation on the environment ${ }^{13}$.

\section{Non Associative Learning}

Non associative learning refers to a relatively permanent change in the strength of response to a single stimulus due to repeated exposure to that stimulus. Learning involving exposure usually to a single event and that is presumed not to reflect learning of a relationship between multiple events. Changes due to such factors as sensory adaptation, fatigue or injury do not qualify as non associative learning. It is not necessary that the animal learns to associate the stimuli involved (thus the name). For example, we learn language by limitation of people who already speak. This involves no association of stimuli and is clearly more complicated than habituation $^{14}$.

\section{Habituation}

Habituation is a form of learning in which an organism decreases or ceases to respond to a stimulus after repeated presentations. Essentially, the organism learns to stop responding to a stimulus which is no longer biologically relevant. For example, organisms may habituate to repeated sudden loud noises when they learn these have no consequences ${ }^{15-16}$.

\section{Sensitization}

Sensitization is a non-associative learning process in which repeated administration of a stimulus results in the progressive amplification of a response. Sensitization often is characterized by an enhancement of response to a whole class of stimuli in addition to the one that is repeated. In sensitization, a stimulus to one pathway enhances reflex strength in another. An example, again taken from experiments in Aplysia and repetition of a painful stimulus may make one more responsive to a loud noise ${ }^{17}$.

\section{TYPES OF MEMORY}

\section{Sensory Memory}

Sensory memory holds sensory information less than one second after an item is perceived. The ability to look at an item and remember what it looked like with just a split second of observation or memorization is the example of sensory memory. It is out of cognitive control and is an automatic response. With very short presentations, participants often report that they seem to "see" more than they can actually report. The first experiments exploring this form of sensory memory were precisely conducted by using the partial report paradig $^{18}$.

Three types of sensory memories exist. Iconic memory is a fast decaying store of visual information; a type of sensory memory that briefly stores an image which has been perceived for a small duration. Echoic memory is a fast decaying store of auditory information, another type of sensory memory that briefly stores sounds that have been perceived for short durations. Haptic memory is a type of sensory memory that represents a database for touch stimuli ${ }^{19}$.

\section{Short Term Memory}

Short term memory is also known as working memory. Short term memory allows recall for a period of several seconds to a minute without rehearsal. Its capacity is also very limited. Short term memory is believed to rely mostly on an acoustic code for storing information, and to a lesser extent a visual code. Confusion with recalling acoustically similar letters rather than visually similar letters implies that the letters were encoded acoustically. Deals with the encoding of written text; thus, while memory of written language may rely on acoustic components, generalizations to all forms of memory cannot be made ${ }^{20}$.

\section{Long Term Memory}

Long term memory can store much larger quantities of information for potentially unlimited duration (sometimes a whole life span). Its capacity is immeasurable. For example, given a random seven digit number we may remember it for only a few seconds before forgetting, suggesting it was stored in our short term memory. On the other hand; we can remember telephone numbers for many years through repetition; this information is said to be stored in long-term memory. While short-term memory encodes information acoustically, long term memory encodes it semantically $^{21}$. Long term memory, on the other hand, is 
maintained by more stable and permanent changes in neural connections widely spread throughout the brain. The hippocampus is essential (for learning new information) to the consolidation of information from short term to long term memory, although it does not seem to store information itself $f^{22}$.

\section{Declarative Memory}

Declarative memory or explicit memory is a memory system that is controlled consciously, intentionally and flexibly. A non associative theory of human long term memory according to which the mind lays down a continuous record of experience (trace column), much like a videotape Declarative memory generally involves some effort and intention and we can employ memory strategies such as mnemonics to recall information it is mediated by the hippocampus and frontal lobes and thus damage to these areas may compromise declarative memory. For example people with damage to the hippocampus have difficulty forming new long term declarative memories, while those with frontal lobe damage may experience deficits in working memory. Such as recall and recognition, in which an individual is fully aware that he or she is being tested. It generally declines with age. Examples include recalling the name of an old friend, remembering a list of items to pick up at the store, remembering information for a test and learning a phone number ${ }^{23}$.

\section{Implicit Memory}

No declarative memory or implicit memories are stored differently depending upon how they are acquired. "Fear conditioning" (training that involves use of fearful stimuli) involves the amygdale. Operant conditioning involves the striatum and cerebellum ${ }^{9}$. For example; eye blink conditioning is disrupted by lesions of the dentate and interpositus nuclei of the cerebellum. Classical conditioning, sensitization and habituation involve the sensory and motor systems involved in producing the motor responses being conditioned. Perhaps surprisingly, certain simple reflexes mediated by the spinal cord can be classically conditioned even after the cord has been surgically isolated from the brain. So, it appears that all regions of the nervous system may be capable of memory storage. Priming is an automatic or unconscious process that can enhance the speed and accuracy of a response as a result of past experience. Different cues (see examples below) prompt the retrieval of memory. Memories are stored as a series of connections that can be activated by different kinds of cues; there is not any single location in the brain associated with a specific memory trace. Priming helps trigger associated concepts or memories, making the retrieval process more efficient ${ }^{24}$.

\section{THE LEARNING CYCLE}

The learning cycle consists of four stages: gather sensory experiences through the sensory, cortices; engage in reflective observation, drawing on the temporal lobe; create new concepts in the prefrontal cortex; and actively test through our motor cortices ${ }^{25}$. The complete cycle of learning arises from the very structure of the brain and results in new and lasting physical connections. Gathering; the first part of the cycle involves gathering information. This step engages the sensory cortices, which receive input from the outside world in the form of vision, hearing, touch, position, smell and taste ${ }^{\mathbf{2 6}}$. Reflection; the second part of the cycle, engages the temporal lobe. During reflection, the brain integrates the sensory information received during the gathering stage. Creation; is the point in the learning cycle at which the learner shifts from receiving and absorbing information to creating knowledge in the form of abstractions such as ideas, plans ,concepts and symbolic representations ${ }^{27}$.

\section{MEMORY PROCESS}

When information comes into our memory system (from sensory input), it needs to be changed into a form that the system can cope with, so that it can be stored. Evidence suggests that this is the principle coding system in short term memory (STM) is acoustic coding. When a person is presented with a list of numbers and letters, they will try to hold them in STM by rehearsing them (verbally). Rehearsal is a verbal process regardless of whether the list of items is presented acoustically (someone reads them out) or visually (on a sheet of paper) ${ }^{28}$.

The principle encoding system in long term memory (LTM) appears to be semantic coding (by meaning). However, information in LTM can also be coded both visually and acoustically. Encoding" refers to the processes involved in the transformation of an event in the world into a representation in your mind/brain. By analogy, imagine that you are writing or speaking to a friend in a secret code: you have a message, and you transform that message into the code with the hopes that your friend can decode it (akin to retrieval). Similarly, memory scientists assume that events are transformed into mental representations by encoding processes. A considerable amount of memory research has examined ways that events are encoded by manipulating the way people process materials during study episodes ${ }^{29}$.

\section{Memory Storage}

This concerns the nature of memory stores, i.e. where the information is stored, how long the memory lasts for (duration), how much can be stored at any time (capacity) and what kind of information is held. The way we store information affects the way we retrieve it. There has been a significant amount of research regarding the differences between STM and LTM. Most adults can store between 5 and 9 items in their $\mathrm{STM}^{\mathbf{3 0}}$.

\section{Memory Retrieval}

This refers to getting information out storage. If we can't remember something, it may be because we are unable to retrieve it. When we are asked to retrieve something from memory, the differences between STM and LTM become very clear ${ }^{28}$. STM is stored and retrieved sequentially. For example; if a group of participants are given a list of words to remember and then asked to recall the fourth word on the list, participants go through the list in the order they heard it in order to retrieve the information. LTM is stored and retrieved by association. This is why you can remember 
what you went upstairs for if you go back to the room where you first thought about it. Organizing information can help aid retrieval. You can organize information in sequences (such as alphabetically by size or by time). Imagine a patient being discharged from hospital whose treatment involved taking various pills at various times, changing their dressing and doing exercises. If the doctor gives these instructions in the order which they must be carried out throughout the day (i.e. in sequence of time), this will help the patient remember them. The next sections are focused on a few of the core concepts in the scientific study of memory. An important idea is that each experience leaves behind some kind of memory trace, a representation of the event, in a person's mind/brain. The exact nature of memory traces has not been specified by memory scientists ${ }^{31}$ but the idea that memory traces exist is useful for explaining memory phenomena. Retrieval then refers to processes involved in reconstructing what occurred at a particular place and time. Memory scientists assume that retrieval processes are always initiated by retrieval cues,

\section{REFERENCES}

1. Byrne JH. Learning and memory in aplysia and other invertebrates. Neurobiol Comparat Cognit 1990:293-315.

2. Kandel ER, Schwartz JH, Jessell TM. Principles of neural science. New York: McGraw-Hill 2000.

3. Kimble GA. Hilgard and Marquis conditioning and learning $2^{\text {nd }}$ Edition. New York: Appleton Century Crofts 1961:570.

4. Neuroscience research centre learning and memory. https://med.uth.edu/nrc/research/learning and memory

5. Roediger HL. Cognitive psychology of memory. Learning and memory: A comprehens referen 2008; 2:1-5.

6. Singh V. An ancient approach but turning into future potential source of therapeutics in Alzheimer's disease. Int Res J Pharm 2015; 6(1):10.

7. Gross R. Psychology: The science of mind and behaviour $6^{\text {th }}$ edition, Hachette UK 2010.

8. Bitterman ME, Menzel R, Fietz A, Schafer S. Classical conditioning of proboscis extension in honeybees (Apis mellifera). J Comparat Psychol 1983; 97:107-119.

9. Clark RE, Squire LR. Classical conditioning and brain system. Th Role Awaren Scien 1998; 280:77-81.

10. Pavlov IP. Conditioned reflexes. London: Oxford University Press 1927:430.

11. Cherry K. The psychology of learning; 2016(4). https://www.verywell.com/learning study guide 2795698

12. Skinner BF. The behaviour of organism: An experimental analysis. Cambridge, Massachusetts: BF Skinner Foundation 1938.

13. Skinner BF. Why we need teaching machines. Harva Educat Rev 1961; 31:377-398.

14. Baddeley AD, Hitch GJ. Working memory. The psychology of learning and motivation. Advan Res Theor 1974; 160:4789.

15. Gagliano M, Renton M, Depczynski M, Mancuso S. Experience teaches plants to learn faster and forget slower in environments where it matters. Oecolog 2014; 175:63-72.

16. Wood DC. Habituation in Stentor: produced by mechanoreceptor channel modification. J Neuroscien 1988; 8(7):2254-8

17. Bell J, Dale M. "Informal learning in the workplace", Department for Education and Employment Research Report No. 134. London, England: Department for Education and Employment 1995. identifiable triggers for retrieval processes and that the experience of remembering occurs when retrieval cues interact with information in memory traces ${ }^{32}$. There must be some information about what you did last night stored in your mind (a memory trace) and your remembrance of the past happens because the retrieval cue interacts with the trace information. This section highlights foundational research and review articles on retrieval processes; beginning with Tulving's influential work on the importance of retrieval cues provides an accessible introduction to the idea that the distinctiveness or uniqueness of retrieval cues is a critical determinant of remembering ${ }^{33}$.

\section{CONCLUSION}

Different types of learning and memory were discussed in the present study. An attempt has been made here to collect all available information about the learning and memory which would enable the researchers to get help during their research.

18. Sperling G. "A model for visual memory tasks". J Hum Fact Ergonom Socie 1963; 5(1):19-31.

19. Carlson NR. Psychology: the science of behavior. Boston, Mass: Allyn \& Bacon; 2010.

20. Conrad R. "Acoustic confusions in immediate memory". Brit J Psychol 1964; 55:75-84

21. Baddeley AD. "The influence of acoustic and semantic similarity on long term memory for word sequences”. Quart J Experiment Psychol 1966; 18(4):302-9.

22. Scoville W.B. and Milner B. "Loss of recent memory after bilateral hippocampal lesions". Journal of Neurology, Neurosurgery and Psychiatry 1957; 20:11-21.

23. Koffka K. Principles of gestalt psychology. New York: Harcourt 1935:485.

24. Wickelgren WA. Context sensitive coding, associative memory and serial order in (speech) behaviour. Psychologic Rev 1969; 76:1-15.

25. Marek EA. Why the learning cycle? J Element Scien Educat 2008; 20(3):63-69.

26. Zull JE. The art of changing the brain, First edition stylus publishing, LLC 22883 Quicksilver Drive Sterling, Virginia 2002.

27. Kolb DA. Experiential learning: Experience as the source of learning and development. Englewood Cliffs, N.J.: PrenticeHall 1984

28. Saul M. Stages of Memory encoding storage and retrieval. Cognit Psychol 2013 www.simplypsychogy.org/memory.html

29. Craik FIM, Tulving E. Depth of processing and the retention of words in episodic memory. J Experiment Psychol 1975; 104:268-294

30. Huppert FA, Piercy M. Normal and abnormal forgetting in organic amnesia: effect of locus of lesion. Cort 1979; 15:385-390.

31. Moscovitch M. Memory: Why the engram is elusive. In Science of memory: Concepts. Edited by Roediger HL, Dudai Y and Fitzpatrick SM. 2007:17-21.

32. Tulving E. Cue-dependent forgetting. Americ Scient 1974; 62(1):74-82.

33. Nairne JS. Modeling distinctiveness: Implications for general memory theory. Distinctiven memo 2006:27-46. 